\title{
Associations between Sleep Disorders and Comfort at End-of-Life: Opportunities for Improvement
}

\author{
Karen J Klingman, $\mathrm{PhD}, \mathrm{RN}^{1}$, Suzanne Sullivan, $\mathrm{PhD}^{2}$ \\ ${ }^{1}$ College of Nursing, SUNY Upstate Medical University, Syracuse, NY, USA \\ ${ }^{2}$ School of Nursing, University at Buffalo, Buffalo, NY, USA
}

\begin{abstract}
Comfort and quality of life (QoL) at the end-of-life (EOL) comprise physical and psychosocial domains. The effects of sleep disorders such as insomnia on comfort-related factors have been widely reported for cancer patients, yet the effect of sleep disorders on comfort may be less well-documented for non-cancer conditions. Quantifying these effects may inform improvements in EOL comfort by treating sleep disorders. The objective of this literature review is to quantify associations between sleep disorders and comfort in non-cancer patients at EOL. A systematic review of the literature identified three articles comprising a total of 239 participants, $88 \%$ males, $83 \%$ with cancer. Comfort-related factors included QoL, dyspnea, rumination, depression, delirium, anxiety, cognitive impairment, and suicidal ideation. The effect of sleep disorders on comfort related factors was substantial. There is a gap in the literature indicating a need for future studies in this area.
\end{abstract}

Sleep Med Res 2018;9(2):110-114

Key Words Sleep wake disorders, Palliative care, Terminal care, Patient comfort.
Received: October 26, 2018 Accepted: November 19, 2018

Correspondence

Karen J Klingman, PhD, RN

College of Nursing,

SUNY Upstate Medical University,

Syracuse, NY, USA

Tel +1-315-464-4276

Fax +1-315-464-5168

E-mail klingmak@upstate.edu

ORCID

Karen J Klingman

https://orcid.org/0000-0003-2114-9464

Suzanne Sullivan

https://orcid.org/0000-0003-3064-6884

\section{INTRODUCTION}

People who are nearing the end-of-life (EOL) state that being free from physical suffering and having emotional well-being are among the most important things contributing to their ability to experience a good death. Comfort and quality of life (QoL) at the EOL comprise both physical and psychosocial domains, and satisfaction with sleep may affect physical comfort and emotional status among older adults [1]. Dissatisfaction with sleep may be due to undiagnosed and untreated sleep disorders such as insomnia, sleep apnea, and restless leg syndrome. Sleep disturbances have been associated with depression, fatigue, and pain in cancer patients and dyspnea, pain, and depression, which are highly prevalent at the EOL, may lead to non-beneficial treatments and burdensome hospitalizations during the final year of life $[1,2]$.

With a prevalence of $10-40 \%$ in the general population, and large proportion undetected $[3,4]$, it follows that sleep disorders are also prevalent at EOL, possibly impacting QoL while dying. The prevalence and impact of sleep disorders in cancer patients has been reported $[1,2]$ however the effects of disordered sleep on comfort at the EOL among individuals who do not have cancer are less clear. Moreover, the rate of US hospice patients with cancer fell to $27 \%$ in 2016, while those with non-cancer diagnoses such as Alzheimer's and related dementias rose [5]. It is imperative, therefore, to understand the effects of sleep disorders on comfort for noncancer patients at EOL. Similarly, associations between sleep and pain are well-understood [68] while associations between sleep and other comfort factors are less well-known. Therefore, the purpose of this review of the literature is to explore and quantify the association between sleep disorder/disturbance and non-pain comfort at EOL among people without cancer, as it may be possible to improve comfort and QoL by addressing sleep quality among individuals 
facing life-limiting illness.

\section{METHODS}

A systematic review of the literature utilizing PubMED, CINAHL, and PsycINFO, was developed with the assistance of a Health Sciences Librarian and guided by the following Medical Subject Heading (MeSH) search strategy:

((“Sleep”[Mesh] OR "Sleep Phase Chronotherapy"[Mesh]) OR "Sleep Wake Disorders"[Mesh]) AND (((((“"end of life"[All Fields] OR "EOL"[All Fields]) OR "Palliative Medicine"[Mesh]) OR "Hospice and Palliative Care Nursing”[Mesh]) OR "Palliative Care"[Mesh]) OR "Hospice Care"[Mesh]) OR “Terminal Care" [Mesh]).

After searching PubMED, the CINAHL and PsycINFO databases were searched utilizing the MeSH terms and keywords. Articles were eligible for inclusion if they were published after 1980, involved human subjects 18 years and older, and were written in English. Articles prior to 1980 were excluded because the first edition of the International Classification of Sleep Disorders was published in 1979 [9]. Original research (excluding case studies), dissertations, brief reports, and poster abstracts were included. A search of gray literature was conducted. Articles were independently reviewed and discussed by the authors. After removal of duplicates, title and abstract were reviewed by both authors to identify studies to retain for full text review. Reference lists of the retained studies were used to identify potential additional resources. Once a complete list of articles for full text review were retained, each title was placed into a Google Scholar search and all citing articles and their reference lists were assessed for relevance to the research question.

The overarching strategy was to identify sources quantitatively describing the effects of disordered sleep on non-pain comfort at EOL for non-cancer patients. The EOL was defined as individuals within one year of dying. This was determined by the presence of a terminal diagnosis, those persons receiving hospice or EOL palliative care, or by performance measures meeting Medicare hospice eligibility criteria measured by Eastern Cooperative Oncology Group, Karnofsky Performance Status (KPS), or Palliative Performance Scale (e.g., KPS score $\leq 40)[1]$.

Research focused exclusively on pain or cancer at the EOL were excluded because they were not the focus of this review. Studies were also excluded if they involved palliative sedation or other medication that would impact sleep. Qualitative studies were excluded because the goal of this review was to quantify the effects of sleep disorders on comfort at EOL.

Characteristics and results of the retained studies were tabulated and relevant information, including study limitations and quality assessment, were transcribed into a matrix. Effect sizes (Cohen's d, Pearson's $r$, and odds ratios) were extracted directly from articles when available. Articles not directly reporting effect sizes were reviewed for numerical results to be used for posthoc effect size determination. Effect sizes were categorized as small, medium, or large according to Cohen's criteria $[10,11]$.

\section{RESULTS}

\section{Search Results}

Titles and abstracts (872) were reviewed according to the stated inclusion and exclusion criteria. Sources were liberally retained for full-text review, yielding 18 sources from which an additional 10 were eliminated following full-text reviews. Of the remaining eight sources, five were eliminated because all study participants: 1) had cancer diagnoses and 2) did not meet the definition of EOL. Three articles [12-14] were included for synthesis and analysis (Fig. 1).

\section{Study Results}

The three retained articles all described prospective descriptive studies following patients through home hospice or inpatient palliative care settings (Table 1). The three studies included $\mathrm{n}=239$ participants, with $88 \%(\mathrm{n}=211)$ male, and $83 \%(\mathrm{n}=$ 199) with cancer. Across the three studies, comfort at the EOL was impacted by degradation in physical symptoms (dyspnea, performance status), psychological symptoms (cognitive impairment, nocturnal rumination, anxiety, depression, delirium, suicidal ideation), and QoL were associated with disordered sleep. Overall, the size of these associations were large per the Cohen criteria [10,11] (Table 1).

In a prospective observational study of veterans in home hospice, depression, delirium, anxiety, cognitive impairment, alcohol abuse, and suicidal ideation (SI) were evaluated every two weeks from enrollment to death, discharge, or study withdrawal [12]. An item from the Confusion Assessment Method was used to determine presence of sleep disorder. The prevalence of all conditions rose from first to last visit, indicating an association between sleep disturbance (SD) and comfort. Effect sizes were not reported, therefore an odds ratio (OR) for presence of SI vs. presence of SD was estimated for the patients who had ever indicated SI during hospice care $(n=12)$. However, the prevalence of SD for all patients at first and last visit were given (19.2\% and 38.4\%), thus it was possible to estimate at what OR SI vs. SD became statistically significant. That prevalence was found to be $25 \%$, which yielded OR [ $95 \%$ confidence interval $(\mathrm{CI})]=3.75(1.06,13.20)$.

In a secondary data analysis of a prospective observational study of veterans in home hospice care, the association between disturbed vs. undisturbed sleep as measured by the Pittsburgh Sleep Quality Index [15] and QoL score, depression worsening, 


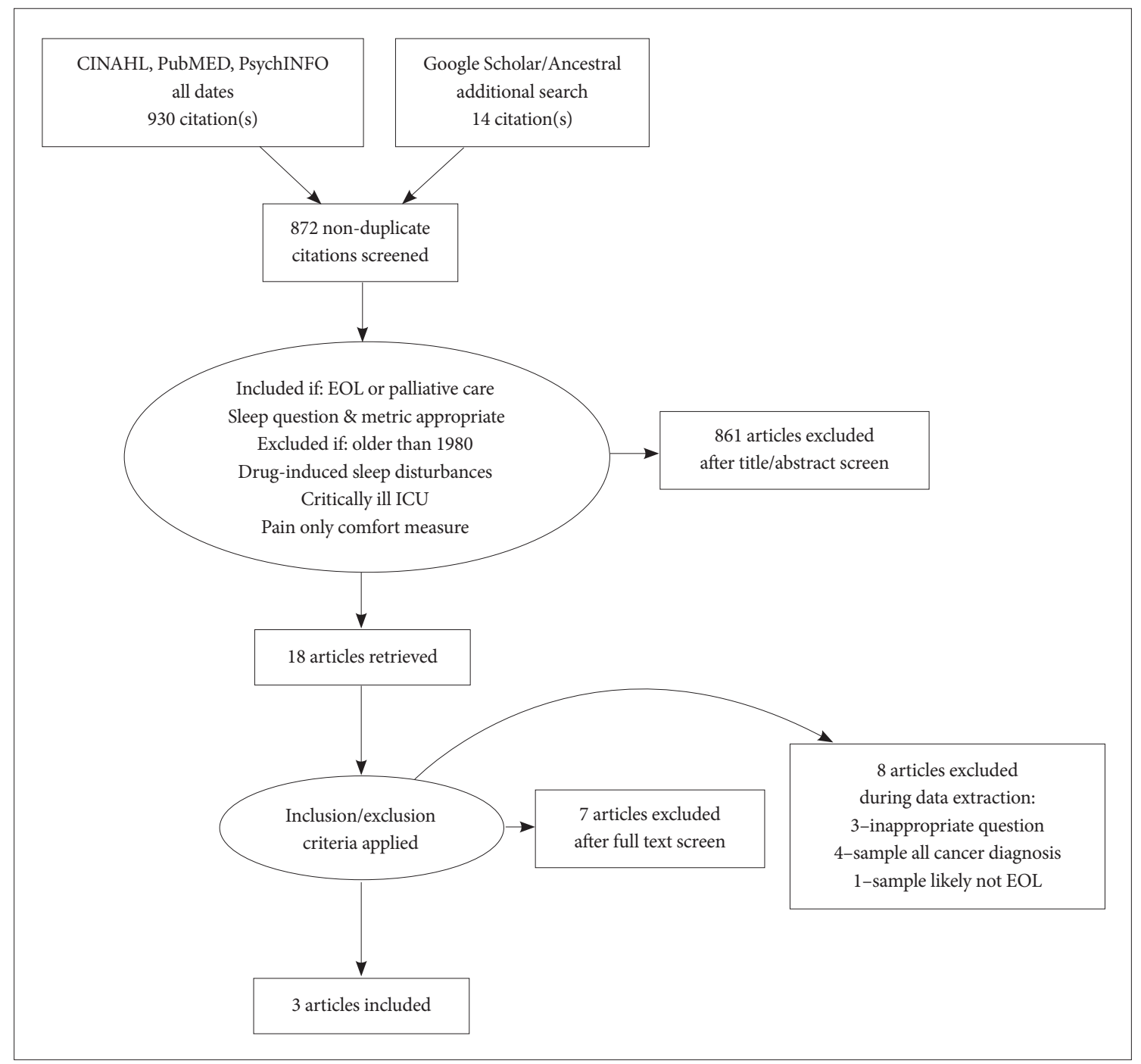

Fig. 1. PRISMA diagram for the effect of sleep disorders on comfort at EOL. EOL: end-of-life.

and onset of delirium during hospice was studied [14]. Quality of life score was rated by patients on a scale from 0 to 100 , with higher numbers indicating better QoL. Effect sizes were calculated post-hoc for this literature review. Cohen's d as determined from QoL scores, number of participants with disturbed and undisturbed sleep, and QoL standard deviations indicates a large effect size of disturbed sleep on non-pain comfort $[\mathrm{d}=$ $1.01,(0.53,1.51)]$. A large effect size $(O R=5.46)$ for being depressed in the last 28 days of life with poor vs. good sleep was determined from Fisher's exact test for prevalence of concomitant depression in the good and poor sleepers (62\% and 23\%, respectively). Confidence intervals around $\mathrm{OR}=5.46$ could not be determined from the data provided. Finally, a hazard ratio [HR of $2.3(1.5,3.7)$ ] for delirium worsening in the last 28 days of life was provided by the authors. When considered alongside the OR results, this HR appeared to be a small-medium effect size; however, this comparison may not be valid, as Cohen $[10,11]$ does not provide guidelines for categorizing HRs as small, medium, or large. The authors also concluded that several parameters did not differ depending on poor vs. good sleep (performance status, depression at any time during hospice, alcohol abuse, cognitive status, anxiety, or suffering score).

In another prospective observational study of palliative care inpatients $(\mathrm{n}=61)$, the differences in clinical, psychological, physical, and environmental factors as a function of insomnia (per investigator-developed one- and three-item scales) was studied [13]. Univariate results indicated insomnia was not associated with physical factors while multivariable logistic regression indicated insomnia was associated with dyspnea. For psychological factors, significant differences were found for depression, anxiety, nocturnal rumination (per the single item insomnia metric) and rumination (per the three-item insomnia metric). Post-hoc calculations for insomnia indicate a medium effect size for depression $[\mathrm{d}=0.75(0.22,1.29)]$ and anxiety $[\mathrm{OR}=3.50(1.19,10.99)]$ and large effect sizes for nocturnal rumination per the single-item metric $[\mathrm{OR}=5.2(1.42,22.75)$ 


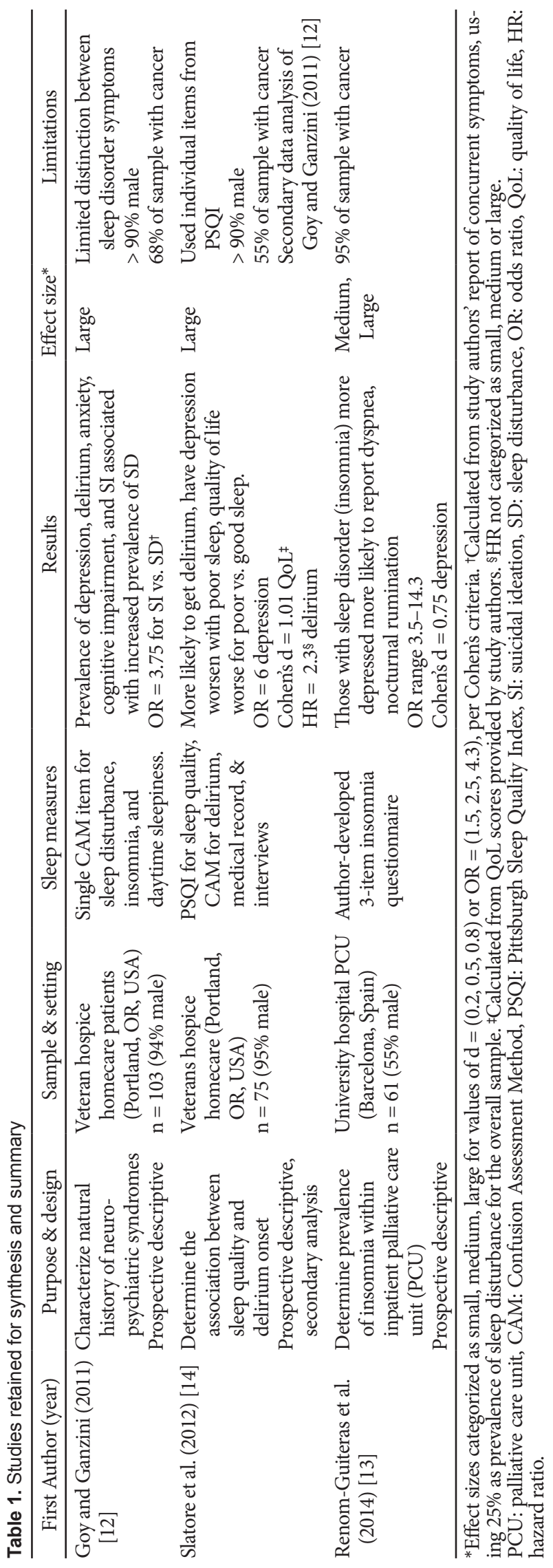

and $6.51(1.51,49.86)$ per the 3-item metric]. Effect sizes were large for physical and psychological factors versus insomnia per the logistic regression. Per the single-item insomnia metric, OR for dyspnea was $7.2(1.65,31.27)$ and OR for nocturnal rumination was $5.5(1.05,28.49)$. Per the 3 -item insomnia metric, OR for rumination was $5.6(1.10,29.1)$.

\section{DISCUSSION}

The purpose of this review of the literature was to explore the association between sleep disorder/disturbance and non-pain comfort at EOL among those individuals who have not been diagnosed with cancer. Despite the rigorous review, and broad body of literature evaluated, there is little evidence describing the relationship between sleep disorders/disturbance and nonpain comfort at the EOL. Based on the large effect sizes found in the available evidence, the findings of this study suggest that disordered sleep has a significant impact on physical and emotional QoL at the EOL. Additionally, for veterans in home hospice care, depression at any time did not differ depending on poor vs. good sleep, the odds of depression worsening during the last 28 days of life were large, and while suffering did not differ depending on poor vs. good sleep, QoL was adversely affected [14].

This review is not without limitations. Despite an exhaustive search of the literature with the assistance of a librarian, only three articles were found meeting the study criteria. In addition, there are no agreed upon definitions describing comfort and sleep disorder/disturbance at EOL. Results are reported in various formats, limiting the ability to categorize effect sizes as small, medium, and high.

Other factors limiting generalizability of the findings include: 1) a dearth of evidence regarding the relationship between disordered sleep and comfort at the EOL, and 2) a substantial overlap in the populations for two of the included studies $[12,14]$. Moreover, despite the attempt to examine non-cancer patients only, no studies excluded cancer altogether. Therefore, individuals with cancer were overrepresented in this study, as were males.

\section{CONCLUSION}

A significant gap exists in the literature for understanding the association between disordered sleep and comfort at the EOL among individuals without cancer. An opportunity exists for EOL and sleep researchers to focus on discovering ways to improve the lives of an expanding and vulnerable population. This type of sleep disorders research is especially important in the hospice and palliative care field because the population of hospice patients is no longer primarily individuals with cancer. 
According to the National Hospice and Palliative Care Organization [5] only $27 \%$ of US hospice patients had primary diagnoses of cancer, and that percentage has been steadily dropping over time. By comparison, cardiac/circulatory and dementia diagnoses each accounted for $18 \%$ of hospice patients. Alleviating pain is certainly a primary goal at EOL, however, alleviating other sources of discomfort, such as fatigue and dyspnea, is also critical. With sleep disorders being diagnosable and treatable conditions, and likely effecting most dimensions of comfort at EOL, knowledge of how sleep disorders affect comfort is critical to guide potential interventions for improving quality of life at EOL. Future work should explore potential interventions for improving non-pain comfort at EOL by improving sleep disorders/disturbances and understanding the reasons and implications of untreated sleep disorder/disturbance on patient wishes for comfort at EOL.

\section{Acknowledgments}

The authors wish to thank Sharon Murphy, Health Sciences Librarian, for her guidance throughout this project.

\section{Conflicts of Interest}

The authors have no financial conflicts of interest.

\section{Authors' Contribution}

Conceptualization: Klingman KJ, Sullivan S. Data curation: Klingman KJ, Sullivan S. Formal analysis: Klingman KJ, Sullivan S. Methodology: Klingman KJ, Sullivan S. Validation: Klingman KJ, Sullivan S. Visualization: Klingman KJ. Writing_original draft: Klingman KJ, Sullivan S. Writing—review \& editing: Klingman KJ, Sullivan S.

\section{REFERENCES}

1. Yennurajalingam S, Bruera E. Oxford American handbook of hospice and palliative medicine and supportive care. 2nd ed. New York: Oxford University Press 2016.
2. Ancoli-Israel S. Sleep disturbances in cancer: a review. Sleep Med Res 2015;6:45-9.

3. American Academy of Sleep Medicine. International classification of sleep disorders. 3rd ed. Darien: American Academy of Sleep Medicine 2014.

4. Ohayon MM. Epidemiological overview of sleep disorders in the general population. Sleep Med Res 2011;2:1-9.

5. National Hospice and Palliative Care Organization (NHPCO). Facts and figures: Hospice care in America [updated 2018; cited 2018 June 21]; Available from: https://www.nhpco.org/sites/default/files/public/ Statistics_Research/2017_Facts_Figures.pdf.

6. Afolalu EF, Ramlee F, Tang NKY. Effects of sleep changes on pain-related health outcomes in the general population: a systematic review of longitudinal studies with exploratory meta-analysis. Sleep Med Rev 2018;39:82-97.

7. Finan PH, Goodin BR, Smith MT. The association of sleep and pain: an update and a path forward. J Pain 2013;14:1539-52.

8. Koffel E, Kroenke K, Bair MJ, Leverty D, Polusny MA, Krebs EE. The bidirectional relationship between sleep complaints and pain: analysis of data from a randomized trial. Health Psychol 2016;35:41-9.

9. Association of Sleep Disorders Centers and the Association for the Psychophysiological Study of Sleep. Diagnostic classification of sleep and arousal disorders. 1979 first edition. Sleep 1979;2:1-154.

10. Cohen J. Statistical power analysis for the behavioral sciences. 2nd ed. New York: Academic Press 1988.

11. Cohen J. A power primer. Psychol Bull 1992;112:155-9.

12. Goy ER, Ganzini L. Prevalence and natural history of neuropsychiatric syndromes in veteran hospice patients. J Pain Symptom Manage 2011; 41:394-401.

13. Renom-Guiteras A, Planas J, Farriols C, Mojal S, Miralles R, Silvent $\mathrm{MA}$, et al. Insomnia among patients with advanced disease during admission in a Palliative Care Unit: a prospective observational study on its frequency and association with psychological, physical and environmental factors. BMC Palliat Care 2014;13:40.

14. Slatore CG, Goy ER, O’hearn DJ, Boudreau EA, O'Malley JP, Peters D, et al. Sleep quality and its association with delirium among veterans enrolled in hospice. Am J Geriatr Psychiatry 2012;20:317-26.

15. Buysse DJ, Reynolds CF 3rd, Monk TH, Berman SR, Kupfer DJ. The Pittsburgh Sleep Quality Index: a new instrument for psychiatric practice and research. Psychiatry Res 1989;28:193-213. 